\title{
Analysis of Morphological Variation of the Knee for Brace Development
}

\author{
James WEBSTER*, Jeremy CORNOLO, Marine MENUT, Katerina KOLLIAS \\ Oxylane Research, Villeneuve-d'Ascq, Lille, France \\ http://dx.doi.org/10.15221/13.335
}

\begin{abstract}
Introduction

Over the past 20 years there has been a significant increase in the participation in recreational and organised sport. Associated with this is the desire to continue training through minor injuries as well as maximise performance with the help of external aids such as knee supports, reducing strain and increasing proprioception. Associated to these types of products are advertised user benefits. However, for these benefits to be upheld it is important that the support applies the correct amount of compression in the appropriate area. Therefore, this study aims to evaluate European knee joint morphology, create separate size divisions and evaluate the morphological variation of the knee within each size through the use of 3D models.
\end{abstract}

\begin{abstract}
Method
1800 scans of European legs (French, Spanish, Portuguese, Germany) were captured using an Artec white light scanner. Principal component analysis was used to identify which of the standard measures used within knee support development should be used to categorise the population. The scans were then analysed through cluster analysis to identify how many sizes were required and how to separate the sizes to obtain $80 \%+$ coverage of the population. Finally, all scans within each size category were used to calculate an average leg, with the centred circumferential standard deviation per $1 \mathrm{~mm}$ to be mapped to create a 3D model of the average leg, average $+2 \mathrm{SD}$ and average -2SD.
\end{abstract}

Results

The PCA results identified that circumferential measure can be used to explain $93 \%$ of the variation within the population, with the measure $8 \mathrm{~cm}$ above knee joint centre having the highest correlation with this factor (0.98). The cluster analysis identified 3 size categories within the data providing $81 \%$ coverage of the European population. An average leg was created for each size, with the standard deviation of each centred circumference calculated. To maximise coverage 2 standard deviations were used to calculate the variation in leg morphology and the location of greatest variation. As the size category was calculated on the $+8 \mathrm{~cm}$ measure the variation in upper leg measures (+8 and $+12 \mathrm{~cm})$ was significantly lower than the knee and lower leg $(-8 \mathrm{~cm})$ measures, $13 \%, 10 \%, 14 \%$ and $15 \%$ respectively.

\section{Conclusion}

The results identify that there is significantly greater variation in calf circumference compared to other anatomical references for knee products. The results identify that through the development of a new sizing system over $80 \%$ coverage could be achieved whilst minimising material elongation to less than $15 \%$ to ensure this is obtained.

\section{Introduction}

Over the past 20 years there has been a significant increase in the participation in recreational and organised sport. Associated with this is the desire to maximise performance as well as to be able to continue training through minor injuries. The knee is one of the key joints sports participants look to protect, with a high percentage of injuries occurring within this region across all mass participation sport, including but not limited to soccer, American football, basketball, rugby and athletics. There are several external aids available on the market for the knee including braces, sleeves, and elastic bandages, all of which can be marketed with different associated user benefits, including increased performance, injury reduction and rehabilitation aid. It is believed that the use of these products facilitate to protect the knee and offer significant performance advantages as a result of underlying mechanisms such as enhanced joint proprioception (Barrett et al, (1991), Birmingham et al. (1998), Jerosch and Prymka (1996), Lephart et al (1992) McNair et al, (1996)). Proprioception has been described in a broader sense than kinesthesis, and it is thought to also include vestibular sensations, inputs from skin, muscles, and joints that are not necessarily perceived (Gandevia, (1996)).

*Email: James.Webster@oxylane.com 
It is apparent that all these benefits are reliant on one crucial factor, the product to be well fitted to prevent movement relative to the body and to apply the correct level of pressure to the skin and in turn the underlying structures. Therefore, this study will focus on the development of a method to develop an accurate and detailed sizing system for knee supports, to help ensure a coverage rate of greater than $80 \%$, as well as using an existing method to create $3 \mathrm{D}$ models of the knee to aid with product design.

\section{Method}

\section{Data Collection}

A scan campaign was completed within Europe across 4 countries (France, Spain, Portugal and Germany), with 1800 adult (16 to 65) scans of the leg (from $15 \mathrm{~cm}$ above the knee to the foot) taken of men and women using an Artec 3D white light scanner. Each individual subject was scanned in a standing position with their leg raised off the ground approximately $35 \mathrm{~cm}$ with a knee angle between 125 and $145^{\circ}$.

\section{Measurement extraction}

The individual scans were treated within Geomagics with 12 anatomical landmarks being identified (figure 1) to enable the leg to be orientated in 3 positions so specific measures could be accurately extracted within Matlab. The 3 orientations were as shown in figure 2 with the origin always located at the knee joint centre. These rotations enabled 4 key measures to be extracted perpendicular to the axis of the leg segment $(12 \mathrm{~cm}$ above knee joint centre $(+12 \mathrm{~cm}), 8 \mathrm{~cm}$ above knee joint centre $(+8 \mathrm{~cm})$, knee joint centre $(\mathrm{KJC}), 8 \mathrm{~cm}$ below knee joint centre $(-8 \mathrm{~cm})$ (figure 3$)$ ). Rotation one was used for $+12 \mathrm{~cm}$ and $+8 \mathrm{~cm}$, rotation 2 for $\mathrm{KJC}$ and rotation 3 for $-8 \mathrm{~cm}$ measurement extraction.

\section{Statistical analysis and sizing system development}

The initial data analysis utilised cooks distance to identify and remove outliers within the data. Once all outliers had been removed from the dataset, Principle component analysis (PCA) was used to determine which measure should be used to segregate the data into different sizes. Finally, the $5^{\text {th }}$ and $95^{\text {th }}$ percentiles were removed before using cluster analysis to identify the number of sizes and how to divide the population into each size based on the principle measure. Once the population had been segregated a minimum and maximum value was calculated for each of the measures, for each size and the population coverage calculated where all measures fell within the defined range.

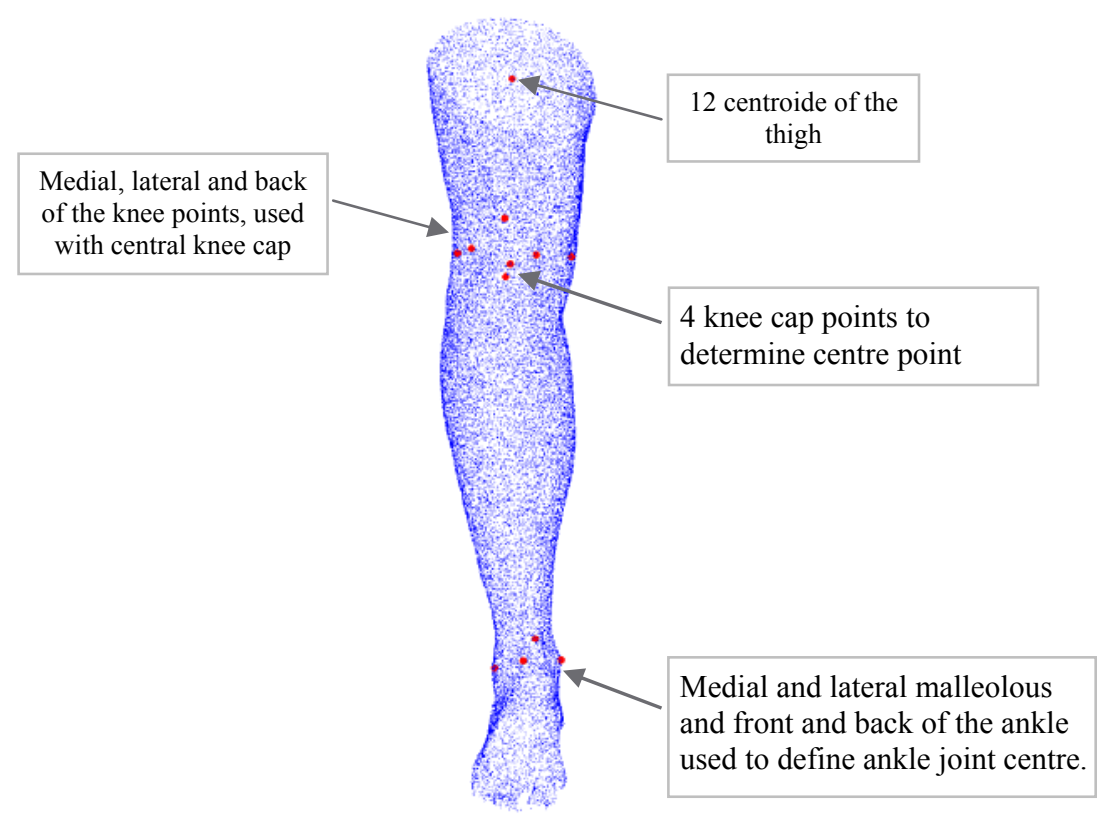

Figure 1: Example point cloud of a scanned leg after treatment within Geomagics, including the 12 anatomical points required for positioning. 

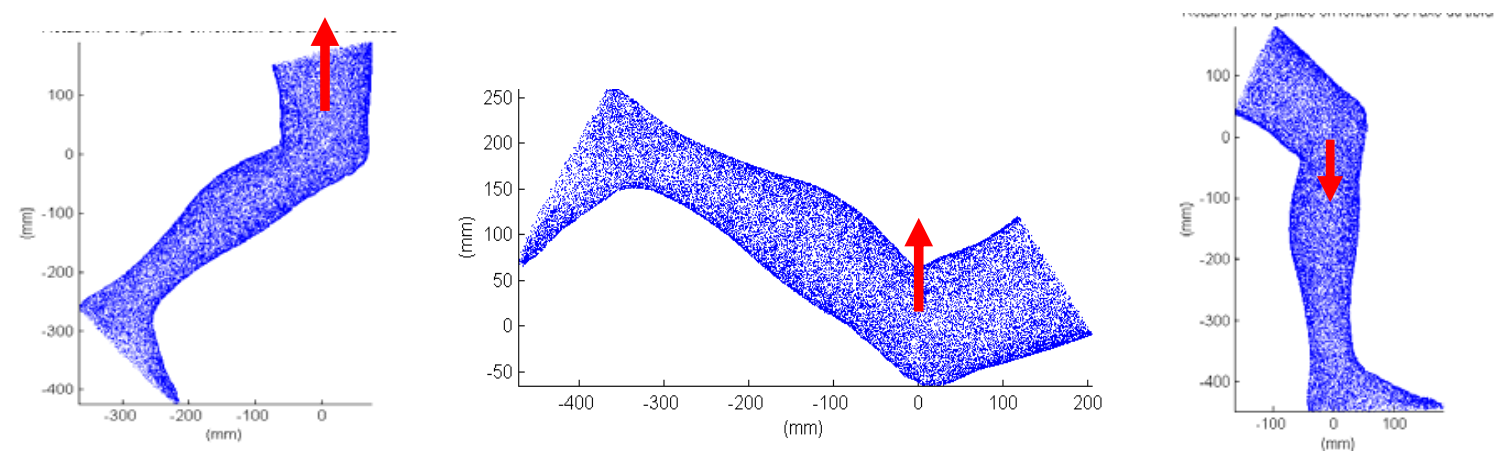

Figure 2: 3 orientations of the leg necessary for data extraction.

\section{Modeling}

The 3D point clouds extracted in Geomagics were separated into groups based on the sizing system created within the previous step. A 3D leg model of the average, average -2 standard deviations and average +2 standard deviations of each centred circumference was created for each size within Matlab through the adaptation of a method developed for the analysis of head sizing within Oxylane research (Kollias, 2012).

The initial step focused on aligning all scans. Initially, 2 point clouds were created for each leg, one was with the thigh orientated in the vertical position and the second with the lower leg vertically orientated. To calculate an average leg using the method developed by Kollias (2012).

Once the average leg for each orientation had been created it was necessary to combine them, using the upper part from orientation one and lower part from orientation 2, as the angle at the knee created significant noise around the non vertically aligned segment. As the origin was always at the knee joint centre a plane was created at the origin to cut the two segments. The parts for the lower and upper part were then combined and exported in STL format. Finally, this process was repeated for +2 standard deviations and -2 standard deviations of each centred circumference, creating maximum, average and minimum leg models for every size.

\section{Results}

The initial analysis focused on the determination of outliers within the data, utilising Cooks distance, with 72 scans being identified as outliers and were therefore excluded from the analysis. Once the outliers had been removed, PCA was used to determine which measure the sizing system should be based on. The results shown within Figure 3 identified that as all measures were circumference based only one factor was required to explain $93 \%$ of the variation. Within this factor all four measures were highly correlated with the variation, but $+8 \mathrm{~cm}$ demonstrated the greatest correlation (0.98) and was utilised as the principle measure.

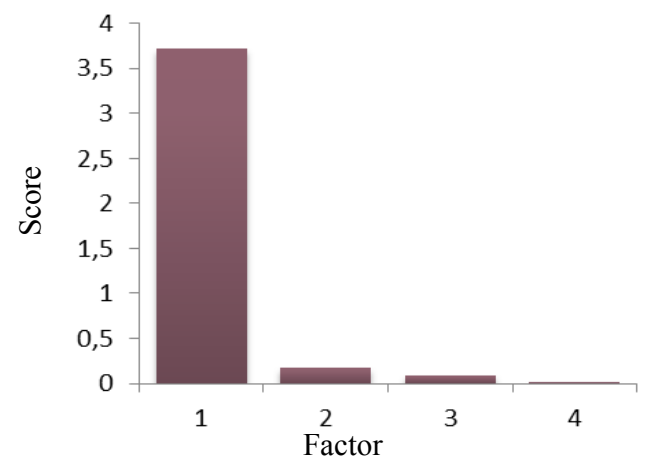

Figure 3: Scree plot of PCA analysis.

Through cluster analysis it was determined that 3 sizes was sufficient, as it would require less than $20 \%$ elongation to cover the full range within a size at all four measurement points, which aligned well with known material characteristics and commercially available products. Utilising 3 sizes as shown within figure 4 , population coverage in terms of perfect fit was $81 \%$ where all 4 measures were within the tolerance of the sizing system. 


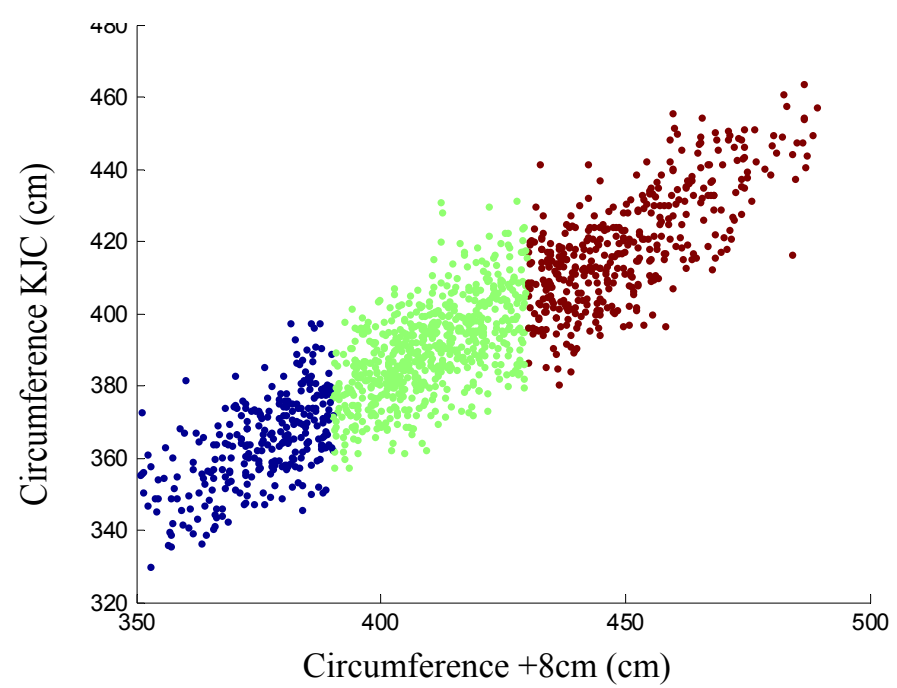

Figure 4: Size separation based on cluster analysis for the $+8 \mathrm{~cm}$ measure.

Finally, 3 models were created for each size, representing the mean (figure 5), +2 standard deviations and -2 standard deviations as shown in figure 6 . To demonstrate the levels of variation at different points of the leg the 4 measures used within the sizing system development were compared across the 3 models. As the size category was calculated on the $+8 \mathrm{~cm}$ measure the variation in upper leg measures $(+8$ and $+12 \mathrm{~cm})$ was lower than the knee and lower leg $(-8 \mathrm{~cm})$ measures, $13 \%, 10 \%, 14 \%$ and $15 \%$ respectively.

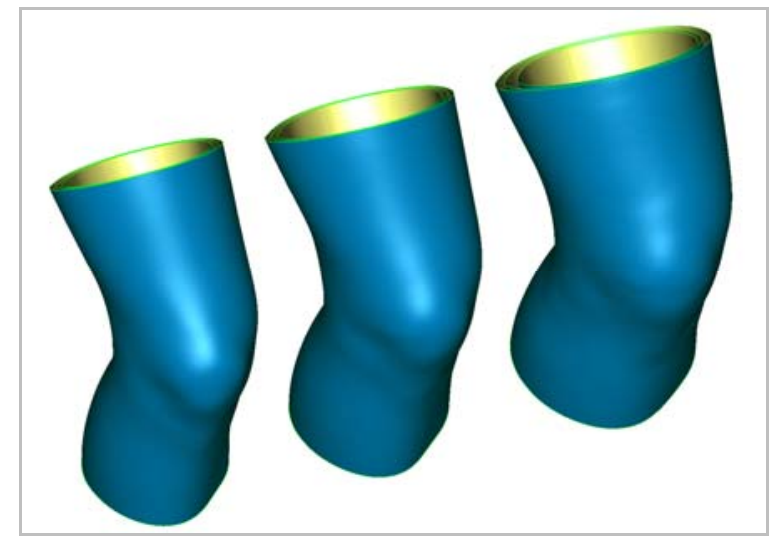

Figure 5: Mean leg model for each of the three sizes.

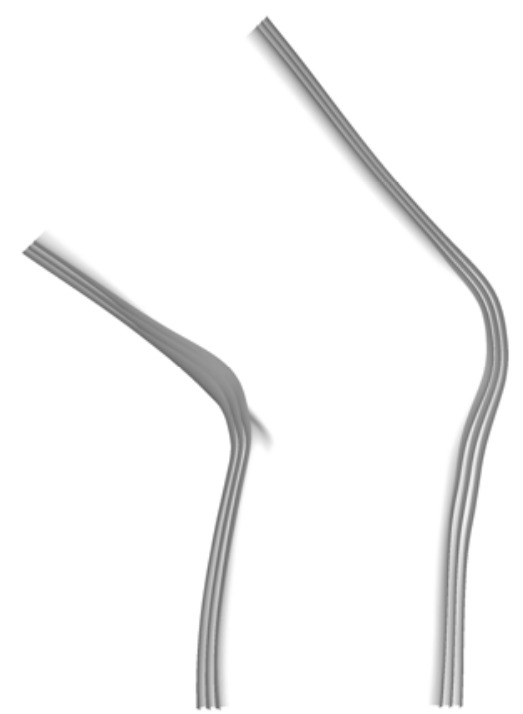

Figure 6: Cross section of the mean, +2 and -2 standard deviation legs for size 1. 


\section{Discussion and conclusions}

This paper details a new method for the development of an accurate and representative sizing system, with the creation of three 3D models utilising the method of Kolias (2012) for use within the product development process for technical products where fit of the product is crucial to ensure advertised user benefits are upheld. The results identified that through the combination of PCA and cluster analysis it was possible to analyse the data obtained through 3D scans, and develop a sizing system representative of the European population with $81 \%$ covered within the category of perfect fit, whilst respecting the known limits of materials typically used within these products. Through the development of this sizing system it was possible to group scans based on size and analyse these with respect to their 3D shape and calculate an average leg. With this method it was also possible to calculate maximum and minimum leg forms for each size representing the change in size and shape across the leg, identifying regions of different variation which could be a significant advancement when considering material placement and choice within products. These 3D models developed in Matlab can be exported in STL or point cloud format and therefore, can be directly imported into most modelling and product development software.

In conclusion, this paper has presented a robust method of sizing system development including the output of detailed 3D models representing the population tested, and enabling degree and area of variation to be shown through the use of 3 models per size, to aid with product development. Future work on this topic should focus on evaluation of the models and the development of a robust validation process to ensure the models are fully representative of all the scans used to create them.

\section{References}

Barrett, D. S., Cobb, A. G. and Bently, G. (1991) Joint Proprioception in normal, osteoarthritic, and replaced knees. J. Bone Joint Surg. 73-B: 53-56

Birmingham, T. B., Kramer, J. F., Inglis, J. T., (1998) Effect of a neoprene sleeve on knee joint position sense during sitting open kinetic chain and supine closed kinetic chain tests. Am. J. Sports Med. 26:562-566.

Jerosch, J. and Prymka. M. (1996) Knee joint proprioception in normal volunteers and patients with anterior cruciate ligament tears, taking special account of the effect of a knee bandage. Arch. Orthop. Trauma Surg. 115:162-166.

Jerosch, J., Prymka, M. and Castro, J.(1996). Proprioception of knee joints with a lesion of the medial meniscus. Acta Orthop. Belg. 62: 41- 45.

Lephart, S. M., Kocher M. S., Fu, F. H. Borsa, P. A. and Harner, C. D. (1992) Proprioception following anterior cruciate ligament reconstruction. J. Sport Rehabil. 1:188-196.

Mcnair, P. J., Stanley, S. N. and Strauss, G. R. (1996) Knee bracing: effects on proprioception. Arch. Phys. Med. Rehabil. 77:287-289.

Perlau, R., Frank, C. and Fick, G. (1995). The effect of elastic bandages on human knee proprioception in the uninjured population. Am. J. Sports Med. 23:251-255.

Gandevia, S. C. (1996) Kinesthesia: roles for afferent signals and motor commands. In: Handbook on Integration of Motor, Circulatory, Respiratory and Metabolic Control during Exercise. L. B. Rowell and J. T. Shepard (Eds.). American Physiological Society, pp. 128-172.

Kollias, Katerina. (2012) Developing 3D sizing system for helmets. Unpublished research. Lille,France: The Author c/o Oxylane Research. 